\section{The postgenomic shipwright}

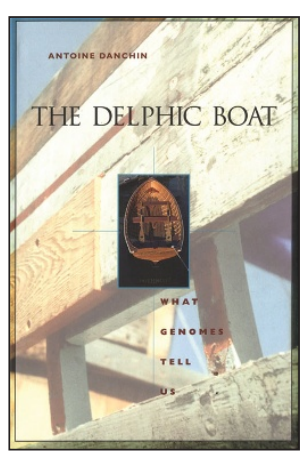

\section{The Delphic Boat: What Genomes Tell Us}

by Antoine Danchin

Alison Quayle, trans.

Harvard University Press, 2003

380 pp. hardcover, $\$ 24.50$

ISBN 0-67400-930-4

\section{Reviewed by Michael R Dietrich}

The Delphic Boat is emblematic of the transition underway in the study of genomes. Genomics initially focused on generating sequence data, but with complete genome sequences available, workers in the field have begun the much more daunting task of understanding the function and regulation of genomes. In this semipopular text, Antoine Danchin mixes history with analysis as he places postgenomic research in the context of a broad overview of molecular and cellular biology.

One might naturally expect Danchin, as a Professor at the Institut Pasteur, to emphasize microbial research. Indeed, his first and most historical chapter offers an account of the development of genomics that focuses on whole-genome projects of microorganisms. The result is a much more nuanced account of genome sequencing than the widely available, triumphal narratives of the human genome project. Danchin's central theme, however, is not the early history of genomics, but rather what lies ahead.

Danchin's concern with functional genomics is expressed by comparing the genome with the Delphic Boat. As a classic riddle of identity, the problem of the Delphic Boat asks if you leave port in a boat and, over the course of your voyage, replace all the individual components of the boat, in what sense do you return to port in the same boat? This riddle focuses our attention on identity as material identity, which is exactly what Danchin wants us to resist. Instead, Danchin refocuses our attention on the relationship between the parts of the genome. Just as the relationship between the planks of the boat are what makes it a functional boat, so Danchin argues that we must understand the relationships that make genomes function in their molecular and cellular contexts.

Michael R. Dietrich is in the Department of Biological Sciences,

Dartmouth College, Hanover, New Hampshire 03755, USA.

e-mail:michael.dietrich@dartmouth.edu
This central analogy of The Delphic Boat allows Danchin to develop an interesting form of antireductionism. Contrasting the material and the symbolic, Danchin advocates a symbolic understanding of fundamental biological relationships. His explication of this important distinction is challenging but essential. Whereas most scientists will readily admit that they represent phenomena with models and concepts, Danchin argues that organisms themselves have this symbolic quality as a property of the interrelation of their parts. This concept of the abstract functional interrelation of the cell allows Danchin to claim that the cell is actually a Turing machine. Turing machines are very basic computational devices that were meant to represent algorithms. A Turing machine reads a tape and carries out an operation according to a set of instructions. The state of the machine, the input from the tape and the set of operational instructions determine the behavior of the Turing machine. Although these machines may be very simple, they can act iteratively to carry out extensive computations. Because a Turing machine may be physically realized in many different ways, this analogy supports Danchin's idea that starting from the bottom up is not going to capture the algorithmic relationships of the cell. That said, Danchin would also like to convince us that cells have the necessary features to act as Turing machines. He argues that processes of compartmentalization, metabolism, memory and manipulation are sufficient, if they are physically separate. Not coincidentally, Danchin defines life with these processes.

According to Danchin, reductionistic attempts to understand cellular function in material (molecular) terms will not capture the fundamental relationships of cells as Turing machines. In terms of genomics, concentrating on the material of the genome (its bases and their sequence) draws our attention away from the interrelations that make function possible. Danchin believes that because sequences have symbolic meaning only in terms of higher-level functions, these interrelations give sequences their biological meaning.

At this point, it may seem that Danchin has wandered far from biology on a philosophical excursion. Indeed he has and does so repeatedly as he addresses conceptual issues, such as the nature of information, complexity, entropy and even life itself. Like all good philosophy of biology, however, Danchin's is grounded in a deep understanding of biology and is worth careful consideration.

The Delphic Boat offers a thoughtful and careful analysis of a great deal of molecular and cellular biology as it relates to genomics. Danchin mentions the ethical implications of genomics and the evolutionary analysis of sequence data, but these are not the true subject of this book. Danchin's contribution lies in his informed and engaging analysis of the challenge of understanding genomes within their functional milieu. 